\title{
Diazepam Metabolism in the Kidneys of Male and Female Rats of Various Strains
}

\author{
Hyung-Sub KIM ${ }^{1) * *}$, Noriaki SAKAI ${ }^{1)}$, Konomu SAITO ${ }^{1)}$, Shoichi FUJITA ${ }^{1)}$ and Mayumi ISHIZUKA ${ }^{1) *}$ \\ ${ }^{1)}$ Laboratory of Toxicology, Department of Environmental Veterinary Sciences, Graduate School of Veterinary Medicine, Hokkaido \\ University, Sapporo 060-0818, Japan
}

(Received 20 March 2009/Accepted 17 August 2009/Published online in J-STAGE 5 November 2009)

\begin{abstract}
Previously, we have reported drastic strain differences of diazepam metabolism in the livers of a variety of rat strain. In this study, to characterize strain and sex differences of diazepam metabolism in the kidney, renal microsomal diazepam metabolic activities were determined in the Dark Agouti (DA), Sprague-Dawley (SD), Brown Norway (BN) and Wistar (WS) strains of rat. We found that the major pathway of diazepam metabolism in the kidney was diazepam $N$-demethylation, which is different from that in the liver, 3 hydroxylation. A Dose-course (12.5-200 $\mu \mathrm{M}$ of diazepam) study revealed that the DA and WS male rats had higher diazepam $N$-demethylation activity than the SD and $\mathrm{BN}$ rats. In contrast to the males, a lower activity of diazepam $N$-demethylation was observed in female BN rats. By Western blot analysis, constitutive protein expressions of cytochrome P450 (CYP) 2C11, which is responsible for diazepam $N$-demethylation, were detected in the 4 strain in both the male and female rats, and the BN rats had lower expression levels of CYP2C11 protein. However, we did not observe significant differences in the kinetic parameters of diazepam $N$-demethylation. Our results suggested that there was a strain difference in CYP-dependent diazepam $N$-demethylation in the rat kidney, which is different from the finding in liver microsomes.
\end{abstract}

KEY WORDS: CYP, diazepam, kidney, rat, strain difference.

Metabolic activities of a number of xenobiotics have been reported to show marked species, strains, sex and age related differences due to their genetic polymorphism of xenobiotics metabolizing enzymes $[5,11,19]$. Diazepam is one of the benzodiazepines widely used in the treatment of anxiety disorders, depression and insomnia. Pathways leading to 3-hydroxy-diazepam (Temazepam) and $\mathrm{N}$-desmethyldiazepam (Nordiazepam) are major in the liver, while $p$ hydroxy-diazepam has been reported to be a minor metabolite in man [3]. Recently, we reported the presence of drastic strain differences in diazepam metabolism in the livers of the Sprague-Dawley (SD), Dark Agouti (DA), Brown Norway (BN) and Wistar (WS) rat strains [20]. SD and BN rats had 300-fold higher diazepam $p$-hydroxylation activity than DA rats at a low concentration of substrate, and 3-fold higher 3-hydroxylation and $N$-demethylation activities in liver of DA rats were observed compared with those of other strains. Although the liver is the main organ responsible for the biotransformation of endogenous and exogenous compounds, other organs, especially the kidney, are involved [1, $2,9]$. Subsequently, we have turned our attentions to the metabolism of diazepam in the kidney.

The major function of the mammalian kidney is to maintain the homeostasis of the internal environment. To achieve this, the kidney is involved in numerous processes including the excretion of xenobiotics. Therefore, it is frequently exposed to potentially toxic compounds, which

\footnotetext{
* Correspondence to: IshizuKa, M., Laboratory of Toxicology, Department of Environmental Veterinary Sciences, Graduate School of Veterinary Medicine, Hokkaido University, Sapporo 060-0818, Japan.

e-mail: ishizum@vetmed.hokudai.ac.jp

**CuRRent address: Kim, H.-S., Clinical Center, National Institute of Health, Bethesda, MD 20852, U.S.A.
}

show a high degree of site-selectivity due to the very complex and heterogeneous anatomical structure of the tissue. Recent studies have shown that several renal cytochrome P450s (CYPs) are inducible and that their levels likely to play a pivotal role in maintenance of renal physiological function and susceptibility to toxic agents [4]. It has been demonstrated that chronic exposure to cadmium impairs the renal CYP-dependnent monooxygenase system through modulating CYP isoforms in WS rats [17]. In the present study, we aimed to reveal the major metabolic pathway of diazepam and its related-CYP expression in the rat kidney. As shown in Fig. 1, diazepam 3-hydroxylation, $p$-hydroxylation and $N$-demethylation are catalyzed by CYP3A2, CYP2D1 and CYP2C11, respectively [13]. A better understanding of the strain and sex differences in the renal CYP isoforms is necessary to understand the role of CYPs in renal physiology and toxicology. Diazepam is one of the typical substrates of CYPs that shows strain and sex differences in rats, and we have previously elucidated the metabolic pathway and strain differences in the livers of rats. In the present study, we focused on the sex and strain differences of diazepam metabolism in the kidney and showed differences between the metabolic pathways of diazepam in the kidneys and livers of a variety of rat strains.

\section{MATERIALS AND METHODS}

Materials: Diazepam and nitrazepam were purchased from Wako Pure Chemical Industries (Osaka, Japan). Diazepam metabolites ( $p$-hydroxy-diazepam, 3-hydroxy-diazepam, $p$-hydroxy- $N$-desmethyl-diazepam, 3-hydroxy- $N$ desmethyl-diazepam and $N$-desmethyl-diazepam) were gifted from Hoffman LaRoche Pharmaceutical Co., Ltd. 


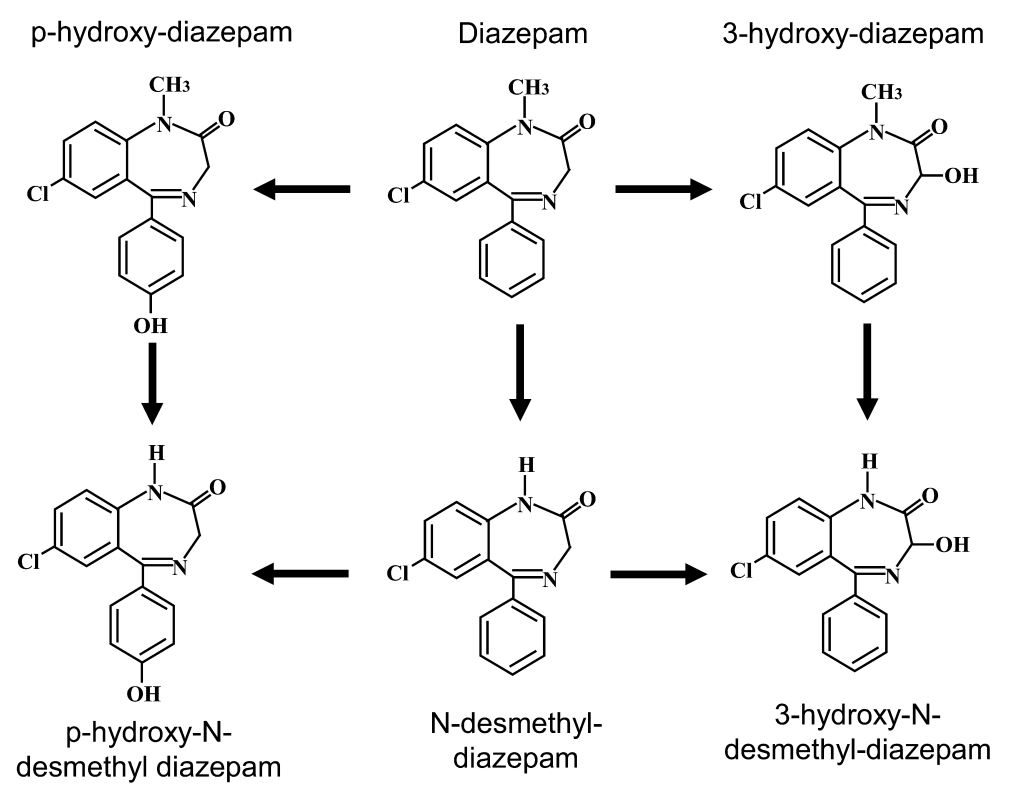

Fig. 1. Metabolic pathway of diazepam in rat renal microsomes. Diazepam 3hydroxylation and $\mathrm{N}$-demethylation are catalyzed by CYP3A2 and CYP2C11, respectively [13].

(Tokyo, Japan). Anti-rat CYP2C11 antibody was obtained from Daiichi Pure Chemicals Co. (Tokyo, Japan). Glucose6-phosphate (G-6-P), glucose-6-phosphate dehydrogenase (G-6-PDH) and $\beta$-nicotinamide-adenine dinucleotide phosphate $(\beta$-NADPH) were obtained from Oriental Yeast Co., Ltd. (Tokyo, Japan). Other chemicals and reagents used were of analytical and biochemical grade.

Animals: Male and female SD, DA, BN and WS rats (10 weeks old) were obtained from Japan SLC, Inc. (Hamamatsu, Japan). All experiments using animals were performed with the supervision and approval of the Institutional Animal Care and Use Committee of Hokkaido University. The rats were housed in plastic cages at $22 \pm 1^{\circ} \mathrm{C}$ with a 12-hr/12-hr light/dark cycle and given laboratory chow and tap water ad libitum.

Preparation of microsomes: Renal microsomes were prepared according to the method of Omura and Sato [15]. Kidney was homogenized in a $0.1 \mathrm{M}$ potassium phosphate buffer ( $\mathrm{pH}$ 7.4) and placed in ice-cold potassium phosphate buffer for preparation of the S-9 fraction. Microsomes were isolated from S-9 fractions by centrifugation at 105,000 $\times \mathrm{g}$ for $70 \mathrm{~min}$ and stored at $-80^{\circ} \mathrm{C}$ until use. The microsomal protein concentration was determined by the method of Lowry et al. [10].

Assay of diazepam metabolism: The reaction mixture (final vol., $0.5 \mathrm{~m} l$ ) contained 12.5 to $200 \mu \mathrm{M}$ of diazepam, 3 $\mathrm{mM} \mathrm{MgCl}$, $5 \mathrm{mM}$ G-6-P and $1.0 \mathrm{mg}$ renal microsomes in $0.1 \mathrm{M}$ potassium phosphate buffer. The reaction was started by adding $1 \mathrm{mM} \beta$-NADPH and 1 unit of G-6-PDH after 3 min of preincubation at $37^{\circ} \mathrm{C}$. After incubation in a water bath for $5 \mathrm{~min}$ at $37^{\circ} \mathrm{C}$, the reaction was stopped by addition of $1.5 \mathrm{ml}$ ethyl acetate. Nitrazepam was added to the mix- ture as an internal standard and was extracted in the organic phase (ethyl acetate) with diazepam and its metabolites by mixing sufficiently. The mixture was centrifuged at $1,000 \times$ $\mathrm{g}$ for $20 \mathrm{~min}$ to separate the aqueous phase. The organic layer was transferred to another tube and evaporated under vacuum, and the residue was dissolved in the HPLC mobile phase. HPLC was performed using a Shimadzu LC-6AV spectrophotometric detector and data processor (Chromatopac C-R6A) and an Inertsil ODS-2 column $(5 \mu \mathrm{m}, 25 \mathrm{~cm} \times$ $4.6 \mathrm{~mm}$ i.d. GL Sciences Inc., Tokyo, Japan). The mobile phase consisted of $0.1 \mathrm{M}$ potassium phosphate buffer $(\mathrm{pH}$ 3.0):acetonitrile:methanol (8:5:4 by vol.) at a flow rate of $0.9 \mathrm{ml} / \mathrm{min}$, and the wavelength for detection of absorbance was set at $230 \mathrm{~nm}$. The kinetic data were estimated using the GraphPad Prism 5 software (San Diego, CA, U.S.A.).

Western blot analysis: The constitutive level of CYP2C11 expression was examined by Western blot analysis. Kidney microsomal protein $(25 \mu \mathrm{g})$ was separated by $10 \%$ sodium dodecyl sulfate- polyacrylamide gel electrophoresis as described by Laemmli et al. [8]. Separated proteins were blotted onto a nitrocellulose membrane and incubated with an anti-CYP2C11 antibody. Goat antimouse IgG was used as the secondary antibody, and the membrane was stained with ECL plus chemiluminescent detection reagent (Amersham, Little Chalfont, U.K.).

Statistical analysis: Curve fitting of enzymatic activities by non-linear regression analysis was performed using GraphPad Prism 5 software. The JMP-IN software (Cary, NC, U.S.A.) was used for statistical analysis. Enzymatic activities among strains were compared using the TukeyKramer HSD test or Dunnett's test, and differences between the sexes were detected using the Student's $t$-test, with a 
Table 1. Diazepam 3-hydroxylation and $N$-demethylation activity in the kidneys of the 4 rat strain. The concentration of substrate (diazepam) was $50 \mu \mathrm{M}$. Other experimental details are described in Materials and Methods

\begin{tabular}{llllll}
\hline & & \multicolumn{1}{c}{ SD } & \multicolumn{1}{c}{ DA } & \multicolumn{1}{c}{ BN } & WS \\
\hline Male & 3-hydroxylation & $6.15 \pm 0.10$ & $6.23 \pm 0.12$ & $6.22 \pm 0.03$ & $6.14 \pm 0.12$ \\
& $N$-demethylation & $15.7 \pm 0.9$ & $19.3 \pm 0.9^{*}$ & $13.8 \pm 0.9$ & $21.3 \pm 1.4^{*}$ \\
\hline Female & 3-hydroxylation & $6.27 \pm 0.31$ & $6.21 \pm 0.13$ & $6.09 \pm 0.06$ & $6.19 \pm 0.32$ \\
& $N$-demethylation & $20.4 \pm 0.9^{* *}$ & $19.7 \pm 0.9$ & $16.6 \pm 2.9^{*}$ & $20.9 \pm 2.5$ \\
\hline
\end{tabular}

The values represent means $\pm \mathrm{sd} .(\mathrm{pmol} / \mathrm{mg}$ protein/min, each $\mathrm{n}=4) . *$ Significantly different from the SD male or female rats $(p<0.05)$. ** Significantly different from the male rats $(p<0.05)$.

$p<0.05$ indicating a significant difference.

\section{RESULTS}

Diazepam metabolism in renal microsomes: Table 1 shows the diazepam metabolic activity in renal microsomes of the various rat strains. At the substrate concentration over $50 \mu \mathrm{M}$ diazepam, the activities of both diazepam $N$ demethylation and 3-hydroxylation were observed in the renal microsomes; however, the activity of diazepam $p$ hydroxylation was not detected. In addition, we did not detect secondary metabolites of diazepam in reaction of renal microsomes. At the substrate concentration of 12.5 $\mu \mathrm{M}$ diazepam, the activity of diazepam $N$-demethylation was detected only, which showed that the primary reaction is diazepam $N$-demethylation, and the diazepam $N$-demethylation activity was 3-4 times higher than that of diazepam 3-hydroxylation at the dose of $50 \mu \mathrm{M}$ diazepam (Table 1). The activity of diazepam $N$-demethylation was markedly higher in microsomes of male DA and WS rats than that of $\mathrm{SD}$ or BN rats, while the activity of diazepam 3-hydroxylation did not show any sex or strain differences. In female rats, significantly low diazepam $N$-demethylation activity was observed in the BN rats compared with the other strains. Diazepam $N$-demethylation was significantly higher in the female SD rats than in the male SD rats, indicating a sex difference.

Kinetic parameters of diazepam metabolism: To confirm the above differences in $N$-demethylation of diazepam, a dose-course study of diazepam metabolism was performed in the incubation mixture containing the NADPH generating system, 12.5-200 $\mu \mathrm{M}$ diazepam and renal microsomes. The mean enzymatic activity of diazepam $N$-demethylation is shown in Fig. 2. In the male rats, the activity of diazepam $N$-demethylation was in the order of $\mathrm{WS}=\mathrm{DA}>\mathrm{SD}=\mathrm{BN}$, and the order of activity was $\mathrm{WS}=\mathrm{DA}=\mathrm{SD}>\mathrm{BN}$ in the female rats. However, there was no significant difference in the kinetic constants of diazepam $N$-demethylation among the $\mathrm{SD}, \mathrm{BN}, \mathrm{DA}$ and WS rats (Table 2).

CYP2C11 expression in renal microsomes: Western blot analysis revealed that the protein expression of CYP2C11, which is responsible for diazepam $N$-demethylation, was observed in the kidneys of all the tested strains of male and female rats (Fig. 3). As shown in Fig. 3, both male and female $\mathrm{BN}$ rats had weaker expression levels of CYP2C11
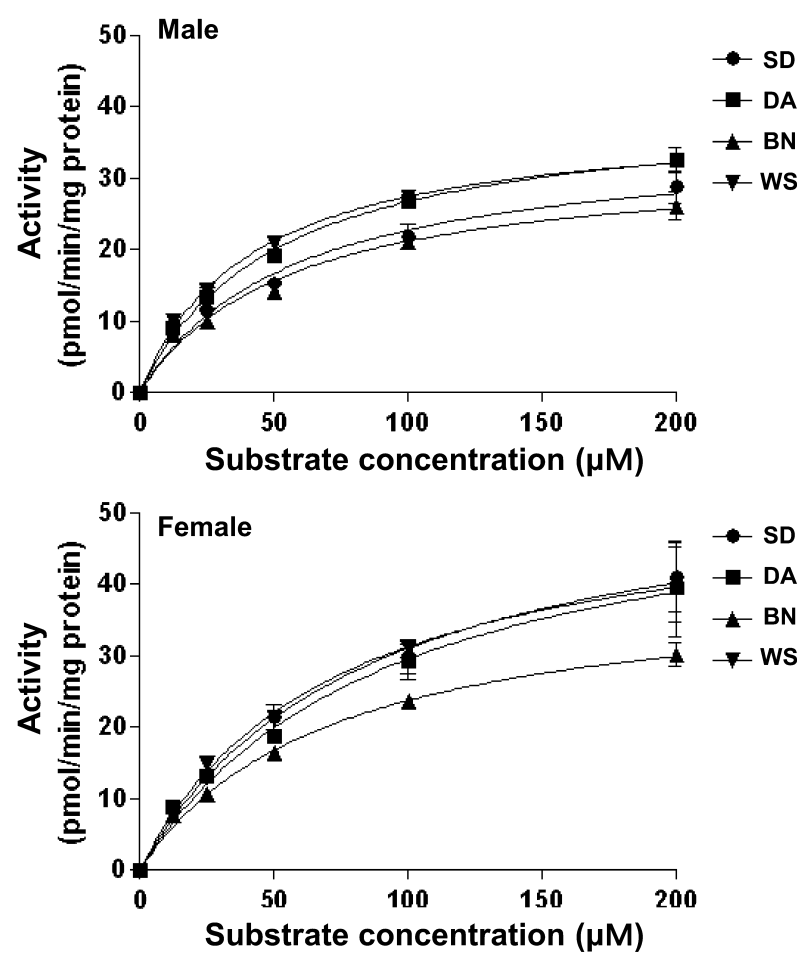

Fig. 2. Effect of substrate concentration on diazepam $N$-demethylation in renal microsomes from adult male and female rats. The concentration range of substrate (diazepam) was 12.5-200 $\mu \mathrm{M}$. Each point represents the average of triplicate data from Sprague-Dawley (SD), Brown Norway (BN), Dark Agouti (DA) and Wistar (WS) strain rats, respectively. Other experimental details are described in Materials and Methods.

than those of WS and DA rats. This result was consistent with the lower diazepam $N$-demethylation activity in the $\mathrm{BN}$ rats among the 4 rat strains measured (Table 1 and Fig. 2). In addition, we did not detect the expression of CYP2C11 in liver microsomes of female rats (data not shown).

\section{DISCUSSION}

The CYP-dependent monooxygenase system plays a crucial role in the biotransformation of not only endogenous, but also exogenous compounds [14, 17]. The monooxygenase activities mediated by the CYP enzymes in rat hepatic 
Table 2. Kinetic parameters for diazepam $N$-demethylation in renal microsomes of male and female rats

\begin{tabular}{llcccc}
\hline & & SD & DA & BN & WS \\
\hline Male & Vmax & $35.82 \pm 2.68$ & $40.44 \pm 1.74$ & $32.84 \pm 2.09$ & $38.79 \pm 0.90$ \\
& Km & $57.52 \pm 10.85$ & $21.27 \pm 5.78$ & $55.01 \pm 9.18$ & $41.41 \pm 2.76$ \\
\hline Female & Vmax & $57.09 \pm 5.75$ & $56.82 \pm 6.77$ & $40.43 \pm 2.21$ & $53.82 \pm 4.68$ \\
& $\mathrm{Km}$ & $84.16 \pm 18.42$ & $92.5 \pm 22.98$ & $70.36 \pm 9.51$ & $72.1 \pm 15.09$ \\
\hline
\end{tabular}

The concentration range of diazepam was 12.5 to $200 \mu \mathrm{M}$. Other experimental details are described in Materials and Methods. Data are expressed as means \pm sd.

$(\mathrm{kDa})$

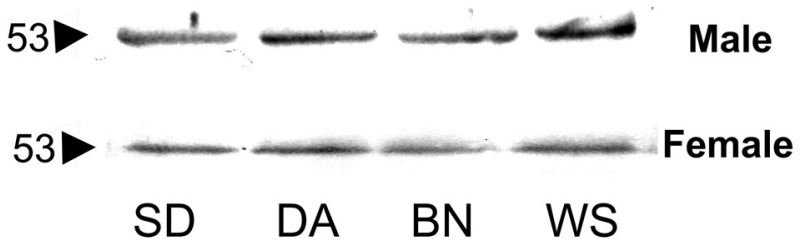

Fig. 3. Western blot analysis of renal CYP2C11 from adult male and female rats of the $\mathrm{SD}, \mathrm{BN}, \mathrm{DA}$, and WS strains using an anti-CYP2C11 antibody. Microsomal protein $(25 \mu \mathrm{g})$ was separated by SDS-PAGE using $10 \%$ acrylamide gel. After transfer onto nitrocellulose membranes, the membranes were incubated with an anti-CYP2C11 antibody. They were washed with a $0.1 \%$ solution of Tween-20 in PBS, incubated for $1 \mathrm{hr}$ with an anti-goat IgG conjugated to horseradish peroxide and stained with ECL reagent.

microsomes have also been detected in renal microsomes [18]. Recognition of the genetic polymorphism in the drugmetabolizing enzymes is important because these differences depending on the strain of the experimental animals may cause significant differences in the results of pharmacokinetic, and pharmacological or toxicological studies [11, 19]. Neville et al. [13] reported that 3-hydroxylation and $N$ demethylation of diazepam proceeds in the hepatic microsomes of WS rats, and that they are catalyzed by CYP isoforms such as CYP3A2 and CYP2C11 (Fig. 1). In the present study, the enzymatic activities in diazepam metabolic pathways were investigated to elucidate strain and sex differences in the kidneys of four rat strains (SD, DA, BN and WS).

Differently from liver microsomes, we did not observed any drastic strain or sex differences in diazepam metabolism in renal microsomes. We detected slight but significant differences in diazepam $N$-demethylation at the substrate concentration of $50 \mu \mathrm{M}$ (Table 2). SD rats showed a sex-related difference in enzyme activity of diazepam $N$-demethylation, while $\mathrm{BN}$ rats had a lower activity of diazepam $\mathrm{N}$-demethylation in both males and females. However, the kinetic parameters of $\mathrm{Km}$ and Vmax showed no significant differences in the range of $12.5 \mu \mathrm{M}$ to $200 \mu \mathrm{M}$ of diazepam. In addition to the diazepam metabolic profiles in the renal microsomes of the four strain, we also found that diazepam $N$-demethylation is the major metabolic pathway in the rat kidney.
As shown in Fig. 3, a high expression of CYPA2C11 was observed in the kidneys of the male and female rats. However, the expression levels of CYP3A2 and CYP2D1 were extremely low compared with CYP2C11 in the renal microsomes, and we did not detect any strain differences in these apoprotein expressions (data not shown). The enzyme activity of diazepam $N$-demethylation corresponded to the expression of CYP2C11 in the renal microsomes of the rats. In the present study, we reported, for the first time, that CYP2C11 was expressed at comparable levels in both male and female rats. Renal CYP2C11 functions in the metabolism of both exogenous chemicals, such as thiophene and cephaloridine, and endogenous chemicals such as testosterone [4]. In addition, CYP2C11 epoxidizes the 11, 12-olefins and 14, 15-olefins, and its expression is induced by high dietary salt administration [22]. The kidney is a rich reservoir of arachidonic acid, which is metabolized by CYP4A and CYP2C gene subfamily isoforms to produce epoxyeicosatrienoic acids (EETs) and 19- and 20-hydroxy-eicosatrienoic acids (19- and 20-HETE). These metabolites have been implicated in a variety of physiologically important processes, including regulation of renal tubular $\mathrm{Na}^{+}$and $\mathrm{K}^{+}$ fluxes, renin activities and water permeability and have been shown to be potent systemic vasodilators and enantioselective intrarenal vasoconstrictors $[7,12]$. In rats, the CYP2C gene subfamily is composed of 8 members, 2 of which are sex-specific (CYP2C11 and 2C12), and CYP2C11 has not been detected in the female liver [16]. CYP2C11, the predominant male-specific CYP isoform, is expressed in the livers of adult rats and mainly regulated by the sexually determined circulation profile of growth hormone, which is modulated by release of testosterone from the testes and by conversion to estradiol in the brain during the neonatal period [6]. However, the present study revealed that female rats showed similar enzyme activity profiles and expression of CYP2C11-recognized protein, indicating that the expression of CYP2C11 in the rat kidney is sex independent. Cells of the proximal convoluted tubule are the first to be exposed to the glomerular ultrafiltrate, and proximal tubular (PT) cells actively transport a wide variety of charged organic and inorganic compounds. Testosterone, which is metabolized by several different CYP isoforms, is $2 \alpha$ - and $16 \beta$-hydroxylated in primary PT cells and kidney microsomes, indicating CYP2C11 activity [21]. However, in contrast to the diazepam $N$-demethylation in the male rat kidney, the enzyme activity of diazepam 3-hydroxylation 
showed no significant differences at $50 \mu \mathrm{M}$ of substrate, and there were no significant differences in the level of CYP3A2 expression in the kidneys of the male rats (data not shown). This observation leads us to consider that enzyme activities related to CYP2C11 may affect the metabolism of diazepam in the kidney and that blood pressure can be regulated by the metabolites.

Moreover, we also found different metabolic pathways of diazepam between the hepatic and renal microsomes of rats. As reported previously, although the activity of diazepam $p$ hydroxylation was high in the livers of SD and BN rats, we could not observe this enzymatic activity in the kidneys of the four rat strains. The dominant metabolic pathway in the liver was diazepam 3-hydroxylation, however, we revealed in the present study that diazepam $N$-demethylation was the major pathway in the kidney.

The sex and strain differences should be noted as physiological information about the xenobiotic-metabolizing enzymes, and the strain differences may affect the results of toxicological studies in rats. The strain and sex differences in diazepam metabolism were observed in the kidneys of the four rat strains; however, the patterns were markedly different from those of livers. In conclusion, the current study demonstrated that the main reaction in the kidney was diazepam $N$-demethylation mediated by CYP2C11 and revealed no enzymatic activity profile of diazepam $p$-hydroxylation in the rat kidney, which is the major metabolic pathway of diazepam in the livers of $\mathrm{SD}$ and $\mathrm{BN}$ rats.

ACKNOWLEDGMENTS. This study was partly supported by Grants-in-Aid for Scientific Research from the Ministry of Education, Culture, Sports, Science and Technology of Japan (No. 1920802807 and No. 19671001).

\section{REFERENCES}

1. Armbrecht, H. J., Lakshmi, V. M., Wickstra, J., Hsu, F. F. and Zenser, T. V. 2007. Metabolism of a heterocyclic amine colon carcinogen in young and old rats. Drug Metab. Dispos. 35: 633-639.

2. Baker, J. R., Satarug, S., Reilly, P. E., Edwards, R. J., Ariyoshi, N., Kamataki, T., Moore, M.R. and Williams, D. J. 2001. Relationships between non-occupational cadmium exposure and expression of nine cytochrome P450 forms in human liver and kidney cortex samples. Biochem. Pharmacol. 62: 713-721.

3. Beischlag, T.V., Kalow, W., Mahon, W.A. and Inaba, T. 1992. Diazepam metabolism by rat and human liver in vitro: inhibition by mephenytoin. Xenobiotica 22: 559-567.

4. Cummings, B.S., Zanger, R.C., Novak, R.F. and Lash, L. H. 1999. Cellular distribution of cytochrome P-450 in the rat kidney. Drug Metab. Dispos. 27: 542-548.

5. Fujita, S., Kitagawa, H., Ishizawa, H., Suzuki, T. and Kiyani, K. 1985. Age-associated alterations in hepatic glutathione-Stransferases. Biochem. Pharmacol. 34: 3891-3894.

6. Ishizuka, M., Yonemoto, J., Zaha, H., Tohyama, C. and Sone, H. 2003. Perinatal exposure to low doses of 2,3,7,8-tetrachlorodibenzo-p-dioxin alters sex-dependent expression of hepatic
CYP2C11. J. Biochem. Mol. Toxicol. 17: 278-285.

7. Korashy, H. M., Elbekai, R. H. and EL-Kadi, A. O. S. 2004. Effects of renal disease on the regulation and expression of renal and hepatic drug-metabolizing enzymes: a review. Xenobiotica 34: 1-29.

8. Laemmli, U. K. 1970. Cleavage of structural proteins during the assembly of the head of bacteriophage T4. Nature 227: 680-685.

9. Lash, L. H., Putt, D. A., Huang, P., Hueni, S. E. and Parker, J. C. 2007. Modulation of hepatic and renal metabolism and toxicity of trichloroethylene and perchloroethylene by alterations in status of cytochrome P450 and glutathione. Toxicology 235: $11-26$.

10. Lowry, O. H., Rosebrough, N. J., Farr, A. L. and Randall, R. J. 1951. Protein measurement with the Folin phenol reagent. $J$. Biol. Chem. 193: 265-275.

11. Maeda, Y., Morita, K., Tasaki, T., Kazusaka, A., Imaoka, S., Funae, Y. and Fujita, S. 1997. Strain differences in age-associated change in testosterone 6-hydroxylation in Wistar and Dark Agouti rats. Environ. Toxicol. Pharm. 3: 1-9.

12. Mcgreff, J. C. 1991. Cytochrome P450 metabolism of arachidonic acid. Annu. Rev. Pharmacol. Toxicol. 31: 339.

13. Neville, C.F., Ninomiya, S., Shimada, N., Kamataki, T., Imaoka, S. and Funae, Y. 1993. Characterization of specific cytochrome P450 enzymes responsible for the metabolism of diazepam in hepatic microsomes of adult male rats. Biochem. Pharmacol. 45: 59-65.

14. Novak, R. F. and Woodcroft, K. J. 2000. The alcohol-inducible form of cytochrome P450 (CYP2E1): role in toxicology and regulation of expression. Arch. Pharm. Res. 23: 267-282.

15. Omura, T. and Sato, S. 1964. The carbon monoxide-binding pigment of liver microsomes. J. Biol. Chem. 239: 2370-2378.

16. Oropeza-Hernandez, L. F., Lopez-Romero, R. and Albores, A. 2003. Hepatic CYP1A, 2B, 2C, 2E and 3A regulation by methoxychlor in male and female rats. Toxicol. Lett. 144: 93-103.

17. Plewka, A., Plewka, D., Nowaczyk, G., Brzoska, M. M., Kaminski, M. and Moniuszko-Jakoniuk, J. 2004. Effect of chronic exposure to cadmium on renal cytochrome P450dependent monooxygenase system in rats. Arch. Toxicol. 78: 194-200.

18. Ronis, M. J., Huang, J., Longo, V., Tindberg, N., IngelmanSundberg, M. and Badger, T. M. 1998. Expression and distribution of cytochrome P450 enzymes in male rat kidney: effects of ethanol, acetone and dietary conditions. Biochem. Pharmacol. 55: 123-129.

19. Saito, K., Kim, H. S., Sakai, N., Ishizuka, M., Kazusaka, A. and Fujita, S. 2004a. Polymorphism in diazepam metabolism in Wistar rats. J. Pharm. Sci. 93: 1271-1278.

20. Saito, K., Sakai, N., Kim, H. S., Ishizuka, M., Kazusaka, A. and Fujita, S. 2004b. Strain difference in diazepam metabolism in Sprague-Dawley, Dark Agouti, Brown Norway and Wistar rats. Drug Metab. Dispos. 32: 959-965.

21. Schaaf, J. S., De-Groene, E. M., Maas, R. F., Commandeur, J. N. M. and Fink-Gremmels, J. 2001. Characterization of biotransformation enzyme activities in primary rat proximal tubular cells. Chem-Biol. Inter. 134: 167-190.

22. Zhao, X., Pollock, D. M., Inscho, E. W., Zeldin, D. C. and Imig, J. D. 2003. Decreased renal cytochrome P4502C enzymes and impaired vasodilation are associated with angiotensin salt-sensitive hypertension. Hypertension 41: 709-714. 